\title{
Dynamic bandwidth allocation with playback buffer stabiliza- tion for QoE-aware adaptive video streaming
}

\author{
Ryuta Sakamoto ${ }^{a)}$ and Ryogo Kubo \\ Department of Electronics and Electrical Engineering, Keio University \\ 3-14-1, Hiyoshi, Kohoku-ku, Yokohama, Kanagawa 223-8522, Japan \\ a)sakamoto.ryuta@kbl.elec.keio.ac.jp
}

\begin{abstract}
The quality of experience (QoE) is an index that evaluates the application-level quality of network services. In adaptive video streaming, stabilization of playback buffer level improves the QoE by avoiding video freezing. Although control-theoretic approaches for stabilizing the buffer level are effective to improve the QoE for a single user, they have not considered user preferences of the QoE or bandwidth allocation for multiple users. This study proposes a dynamic bandwidth allocation method that satisfies the QoE requirements of multiple users with different preferences. Simulation results show that the proposed method increases the number of satisfied users and QoE fairness for prioritized users at the expense of the average QoE for all users.
\end{abstract}

Keywords: adaptive video streaming, MPEG-DASH, quality of experience, fairness, playback buffer, bandwidth allocation

Classification: Network

\section{References}

[1] Cisco, "Cisco visual networking index: Global mobile data traffic forecast update, 2017-2022," Feb. 2019.

[2] T. Kimura, T. Kimura, A. Matsumoto, and K. Yamagishi, "Balancing quality of experience and traffic volume in adaptive bitrate streaming," IEEE Access, vol. 9, pp. 15530-15547, Jan. 2021. DOI:10.1109/ACCESS. 2021.3052552

[3] A. A. Barakabitze, N. Barman, A. Ahmad, S. Zadtootaghaj, L. Sun, M. G. Martini, and L. Atzori, "QoE management of multimedia streaming services in future networks: A tutorial and survey," IEEE Commun. Surveys Tuts., vol. 22, no. 1, pp. 526-565, First quarter 2020. DOI:10.1109/COMST.2019.2958784

[4] H. Mao, R. Netravali, and M. Alizadeh, "Neural adaptive video streaming with Pensieve," Proc. SIGCOMM 2017, pp. 197-210, Aug. 2017. DOI: $10.1145 / 3098822.3098843$

[5] X. Yin, A. Jindal, V. Sekar, and B. Sinopoli, "A control-theoretic approach for dynamic adaptive video streaming over HTTP," Proc. SIGCOMM 2015, pp. 325-338, Aug. 2015. DOI:10.1145/2785956.2787486 
video streaming," IEEE Trans. Broadcast., vol. 64, no. 2, pp. 590-601, Jan. 2018. DOI:10.1109/TBC.2018.2789580

[7] R. Sakamoto, T. Shobudani, R. Hotchi, and R. Kubo, "QoE-aware stable adaptive video streaming using proportional-derivative controller for MPEG-DASH," IEICE Trans. Commun., vol. E104-B, no. 3, pp. 286-294, Mar. 2021. DOI:10.1587/transcom.2020EBP3038

\section{Introduction}

Internet video traffic has been increasing in recent years [1]. Adaptive bitrate (ABR) streaming such as MPEG-dynamic adaptive streaming over HTTP (MPEG-DASH) is widely utilized to achieve efficient video streaming services $[2,3]$. The quality of experience (QoE) is an index to evaluate users' satisfaction level in network services. To maintain a certain level of the QoE in video streaming, appropriate bitrate control according to network conditions is a key technique. Recent trends in ABR control include machine-learning [4] and control-theoretic [5] approaches. The control-theoretic approaches have the advantage of being able to design control parameters based on the theoretical analysis of buffer dynamics.

Huang et al. [6] proposed a control-theoretic approach using a proportionalderivative-integral (PID) controller to ensure that the remaining playable time of video data in the playback buffer, which is called buffer level, was stabilized to be a constant. Although this method could prevent video freezing, the bitrate was updated more frequently to maintain a constant buffer level. This could lead to QoE degradation owing to bitrate fluctuations. To address this issue, a mitigation method for the bitrate fluctuations was proposed in [7]. While this method improved the QoE for a single user, it did not consider user preferences of the QoE or bandwidth allocation for multiple users.

This study proposes a dynamic bandwidth allocation method for MPEGDASH users with different QoE preferences. In the proposed system, a proportional-derivative (PD)-based ABR controller [7] is utilized to stabilize the playback buffer level. In addition, the proposed dynamic bandwidth allocation method based on the prioritization using predetermined QoE preferences is installed into a bottleneck link to increase the number of satisfied users and QoE fairness for prioritized users. Simulation results show the effectiveness of the proposed method.

\section{System configuration and playback buffer stabilization}

In this study, we consider the video streaming system for MPEG-DASH shown in Fig. 1, where $C_{\text {available }}$ and $n_{\text {total }}$ denote the available bandwidth and total number of users, respectively. Each DASH client determines a video bitrate to be downloaded for the next segment according to network conditions and requests it to a DASH server. Then, the DASH server sends the requested segment to the DASH client. The video streaming provider has 
information on the users' QoE preferences described in the following section. The network management server allocates the bandwidth of the bottleneck link to each user based on the QoE preferences.

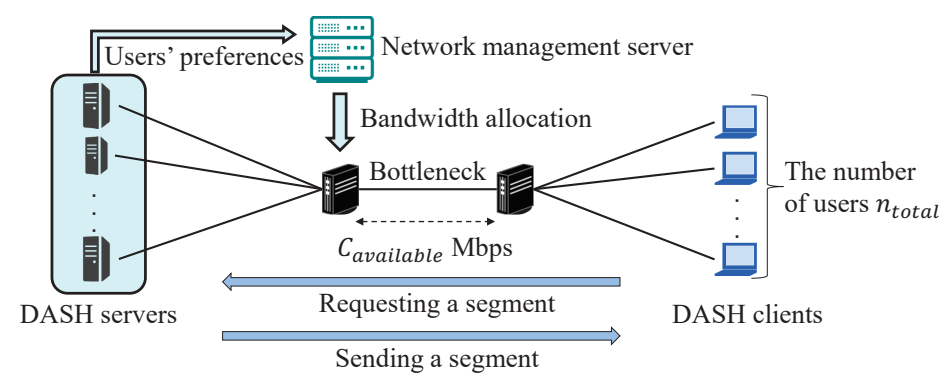

Fig. 1. Video streaming with bandwidth allocation.

In ABR control, the PD-based controller with the mitigation method of bitrate fluctuations [7] is adopted. This method can suppress interruptions and bitrate fluctuations that occur while viewing videos. If the buffer level $b_{k}$ at the start of the $k$-th segment download is less than the lower threshold $B_{t h}$, the target buffer level $b_{k}^{c m d}$ input to the feedback controller is set to $B_{t h}$. On the other hand, if $b_{k}$ is greater than the upper threshold $U_{t h}, b_{k}^{c m d}$ is set to $U_{t h}$. Following the calculation of $b_{k}^{c m d}$, the feedback controller calculates the preset bitrate $r_{k}^{\prime}$ as (1)

$$
r_{k}^{\prime}=-r_{k-1} C(z)\left(b_{k}^{c m d}-b_{k}\right)+r_{k-1},
$$

where $C(z)$ denotes a digital implementation of the PD controller. The bitrate $r_{k}$ to be requested is selected from the bitrate candidates prepared in the DASH server by using a mid-tread quantizer. It is noted that the decrement of $r_{k}$ from the previous segment is limited to $n_{t h}$ quantized steps. If $b_{k}$ ranges from $B_{t h}$ to $U_{t h}$, the bitrate $r_{k}$ is selected based on the throughput. If the buffer level increases, the bitrate candidate which is smaller than and closest to the estimated throughput is selected as $r_{k}$. If the buffer level decreases, the bitrate candidate which is larger than and closest to the estimated throughput is selected as $r_{k}$. It is noted that the decrement of $r_{k}$ from the previous segment is limited to one quantized step.

\section{QoE-aware dynamic bandwidth allocation}

Our proposed bandwidth allocation method utilizes the following QoE model. The QoE for video streaming is significantly affected by three factors, i.e., the bitrate, video freezing, and bitrate fluctuation. By referring to $[4,5,6]$, the total QoE value of user $n$ for segment $k, Q_{n, k}$, is defined as (2)

$$
Q_{n, k}=\alpha_{n} Q_{k}^{v}+\beta_{n} Q_{k}^{f}+\gamma_{n} Q_{k}^{s},
$$

where $Q_{k}^{v}, Q_{k}^{f}, Q_{k}^{s}$ denote the QoE values related to the bitrate, freezing time, and bitrate fluctuation of the $k$-th segment, respectively. In addition, the coefficient $\alpha_{n}, \beta_{n}$, and $\gamma_{n}$ indicate user preferences for the bitrate, freezing 
time, and bitrate fluctuation, respectively. The QoE values $Q_{k}^{v}, Q_{k}^{f}$, and $Q_{k}^{s}$ are calculated as $Q_{k}^{v}=\ln r_{k}, Q_{k}^{f}=-T_{k}$, and $Q_{k}^{s}=-\frac{\left|r_{k}-r_{k-1}\right|}{r_{k}}$, respectively. In the calculation of $Q_{k}^{f}, T_{k}$ denotes the freezing time while viewing the video due to the download process of the $k$-th segment.

The algorithm for the proposed dynamic bandwidth allocation method is shown in Fig. 2. This algorithm is executed in each bandwidth allocation cycle. The proposed method consists of three constituent algorithms, i.e., operations 1-3.

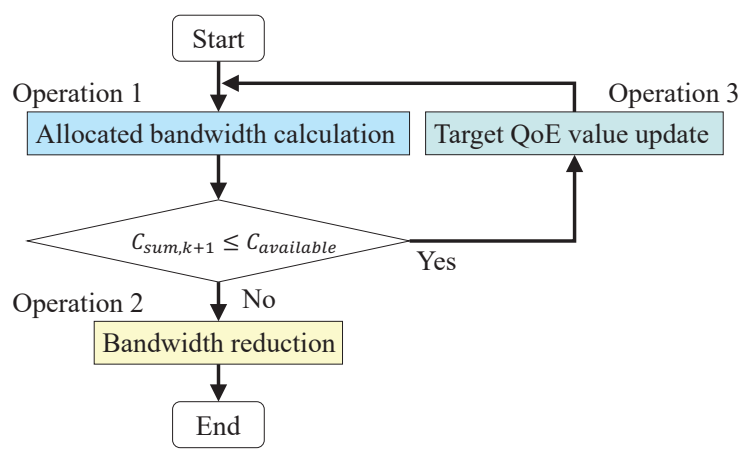

Fig. 2. Algorithm of the proposed method.

Operation 1 calculates the tentatively allocated bandwidth for each user, $C_{n, k+1}$, and their summation for all users, $C_{\text {sum }, k+1}$. Assuming the PDbased ABR controller can achieve stable buffer level control and there are no freezing time and fewer bitrate fluctuations, $Q_{n, k}$ is ideally considered as (3)

$$
Q_{n, k}=\alpha_{n} Q_{k}^{v}=\alpha_{n} \ln r_{k}
$$

This novel assumption for bandwidth allocation is valid only if the PD-based ABR controller [7] is installed. The bitrate $r_{n, k+1}^{r e q}$ required to achieve the target $\mathrm{QoE} Q^{c m d}$ for downloading the $(k+1)$-th segment is estimated as $(4)$

$$
r_{n, k+1}^{r e q}=\exp \left(\frac{Q^{c m d}}{\alpha_{n}}\right) .
$$

The coefficient $\alpha_{n}$ is obtained from users in advance by using machine learning, user setting, or contract. The bandwidth allocated to user $n, C_{n, k+1}$, is set to $r_{n, k+1}^{r e q}$ in the range from $C_{\min }$ to $C_{\max }$. It is noted that $C_{\min }$ and $C_{\max }$ are the minimum and maximum bitrates of the video. If $r_{n, k+1}^{r e q}$ is less than $C_{\text {min }}, C_{n, k+1}$ is set to $C_{\text {min }}$. If $r_{n, k+1}^{r e q}$ is greater than $C_{m a x}, C_{n, k+1}$ is set to $C_{\max }$. Then, if the total allocated bandwidth $C_{\text {sum }, k+1}$ is greater than the available bandwidth $C_{\text {available }}$, the process goes to operation 2. If $C_{\text {sum }, k+1}$ is less than or equal to $C_{\text {available }}$, the process goes to operation 3 .

Operation 2 reduces the bandwidth when the total allocated bandwidth exceeds the available bandwidth. This operation preferentially reduces the bandwidth for the users that require the maximum bandwidth to satisfy their target QoE value, thereby satisfying a relatively large number of users. It is noted that all the users can utilize the bandwidth of at least $C_{\min }$. The 
number of satisfied users can thus be increased at the expense of a few users by repeating this operation. This operation is terminated when $C_{s u m, k+1}$ is less than or equal to $C_{\text {available }}$.

Operation 3 updates the target QoE value when the total allocated bandwidth falls below the available bandwidth just after operation 1 . The target QoE value $Q^{c m d}$ is increased by $A$ to effectively utilize the residual bandwidth and improve the QoE. If the value of $A$ is small, the total allocated bandwidth can be increased gradually, thereby preventing operation 2 from reducing the bandwidth of several users; however, the number of calculations for the allocated bandwidth increases. Therefore, there is a trade-off relationship between the value of $A$ and the calculation frequency of the allocated bandwidth.

\section{Numerical simulation}

The numerical simulations were performed to compare the proposed method and a static bandwidth allocation method, which allocates the available bandwidth equally for all users. In the simulations, the available bandwidth

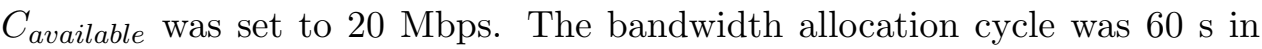
the proposed method. Network delays were neglected since they were much smaller than the bandwidth allocation cycle. The playback duration time per segment $\tau$, playback buffer size $b_{l i m}, B_{t h}, U_{t h}, n_{t h}$, and $A$ were set to $4 \mathrm{~s}$, $50 \mathrm{~s}, \frac{1}{5} b_{\text {lim }}, \frac{4}{5} b_{\text {lim }}, 2$, and 0.01 , respectively. The proportional and derivative gains of the PD controller were set to 0.17 and 0.85 , respectively. Ten video bitrates of 235, 375, 560, 750, 1050, 1400, 1750, 2350, 3600, and $4500 \mathrm{kbps}$ were available on the server.

The total QoE value calculated by (2) was utilized for the analysis to confirm the effect of the bandwidth allocation methods on the ABR controller and the validity of the proposed algorithm based on (3). The user preferences were set randomly within defined ranges, such as $0.8 \leq \alpha_{n} \leq 1.2,4 \leq$ $\beta_{n} \leq 12$, and $2.5 \leq \gamma_{n} \leq 7.5$. These values were obtained by assuming the general preferences listed in $[6]$ to be equal to the median values of the respective parameters. Ten simulations were performed for each case that had a unique number of users, i.e., 5, 10, 15, or 20. The average values and standard deviations of the results of these ten trials were used to perform the analysis. The fairness of the total QoE value was evaluated by calculating Jain's fairness index (FI) for the time-averaged total QoE value of each user. A user with a time-averaged total QoE of $0.96 \times Q^{c m d}$ or more is defined as a satisfied user. The target $\mathrm{QoE}$ value $Q^{\mathrm{cmd}}$ was set to 7.5. A user whose bandwidth was not reduced in operation 2 is defined as a prioritized user. In the static bandwidth allocation, all users are the prioritized users.

The simulation results are shown in Fig. 3. Figs. 3(a)-3(d) demonstrate the ratio of the satisfied users to $n_{\text {total }}$, the FI for the total QoE values of the prioritized users, the ratio of the prioritized users to $n_{\text {total }}$, and the average total QoE values for all the users, respectively. It was confirmed that the proposed method improved the number of satisfied users and the QoE fairness 
for the prioritized users compared to the static bandwidth allocation method, at the expense of the average QoE for all the users. The improvement for the ratio of the satisfied users was remarkable for a large $n_{\text {total }}$ because the proposed method allocated the bandwidth by considering QoE preferences. However, the proposed method generated a few dissatisfied users, resulting in the degradation of the average QoE for all the users. In addition, the freezing time and frequency of bitrate fluctuations were almost the same even if $n_{\text {total }}$ increased because the PD-based ABR controller could stabilize the playback buffer level based on effective throughput. It is noted that low-priority users could experience video freezing because of the bandwidth reduction.

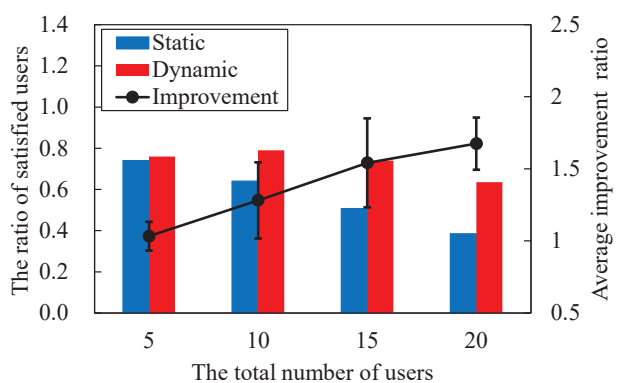

(a) The number of satisfied users

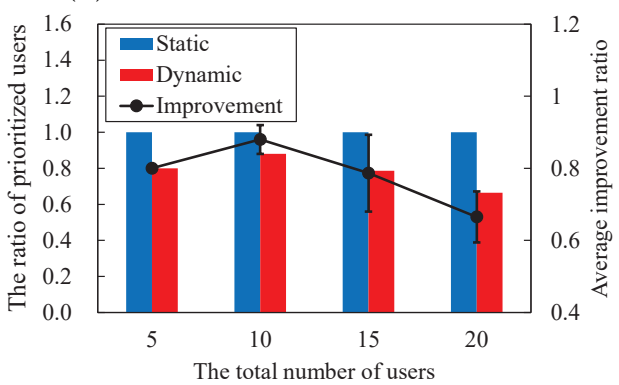

(c) The number of prioritized users

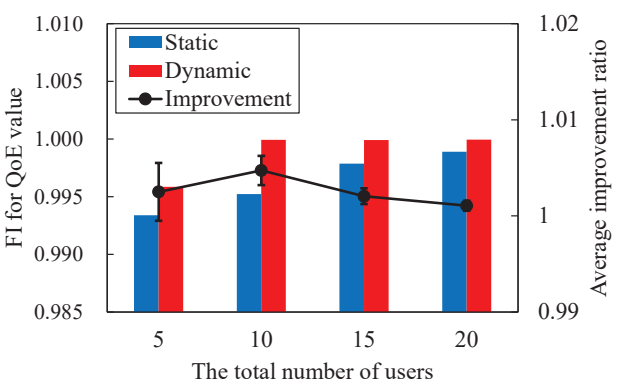

(b) QoE fairness for prioritized users

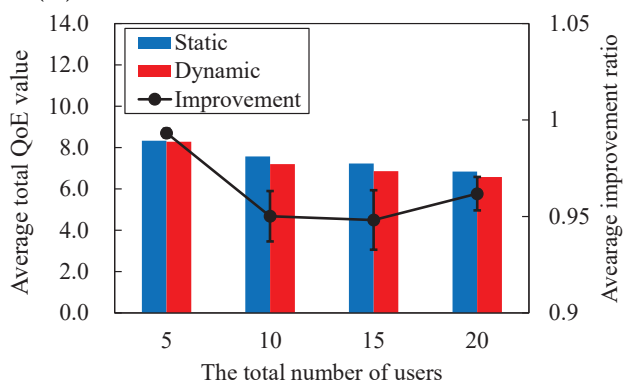

(d) Average QoE for all users

Fig. 3. Simulation results.

\section{Conclusion}

This letter proposed the dynamic bandwidth allocation method to satisfy the QoE requirements of multiple users with different user preferences. The simulation results demonstrated that the proposed method could increase the number of satisfied users and QoE fairness for prioritized users, at the expense of the average QoE for all users.

\section{Acknowledgments}

This work was supported in part by JSPS KAKENHI (Grant Numbers 18H03236 and $18 \mathrm{~K} 11275)$. 